\title{
Discontinuation of proton pump inhibitor use reduces the number of endoscopic procedures required for resolution of walled-off pancreatic necrosis
}

\author{
Patrick C. Powers, Ali Siddiqui ${ }^{1}$, Reem Z. Sharaiha ${ }^{2}$, Grace Yang ${ }^{1}$, Enad Dawod ${ }^{2}$, Aleksey A. Novikov ${ }^{2}$, \\ Amy Javia ${ }^{1}$, Cynthia Edirisuriya ${ }^{1}$, Arish Noor ${ }^{1}$, Tayebah Mumtaz ${ }^{1}$, Usama Iqbal ${ }^{1}$, David E. Loren ${ }^{1}$, \\ Thomas E. Kowalski ${ }^{1}$, Natalie Cosgrove ${ }^{1}$, Yordano Alicea ${ }^{3}$, Amy Tyberg ${ }^{2}$, Iman Andalib ${ }^{2}$, Michel Kahaleh², \\ Douglas G. Adler \\ Department of Internal Medicine, Division of Gastroenterology and Hepatology, University of Utah, Salt Lake City, UT, \\ ${ }^{1}$ Department of Internal Medicine, Division of Gastroenterology and Hepatology, Thomas Jefferson University, ${ }^{3}$ Department \\ of Internal Medicine, Drexel University, Philadelphia, PA, ${ }^{2}$ Department of Internal Medicine, Division of Gastroenterology \\ and Hepatology, Weill Cornell Medicine, New York, NY, USA
}

\begin{abstract}
Background and Objectives: Endoscopic drainage/debridement of symptomatic walled off necrosis (WON) using lumen-apposing metal stents (LAMS) is both safe and effective. While endoscopic management of WON is the standard approach to treatment, the ideal concomitant medical therapy remains unclear. The purpose of this study was to further elucidate the effect of proton pump inhibitor (PPIs) therapy on the technical and clinical success of endoscopic treatment of WON. Methods: Two hundred and seventy-two patients in 8 centers with WON managed by endoscopic drainage using LAMS were evaluated. Patients were followed for at least 6 months following treatment. The patients were divided into two groups: Those that used PPIs continuously during the therapy and those not on PPIs continuously during the interval of therapy. Outcomes included but were not limited to technical success, clinical success, number of procedures performed, and adverse events. Results: From 2013 to 2016, 272 patients underwent WON drainage with successful transmural LAMS placement. The two groups were split evenly into PPI users and non-PPI users, and matched in regards to demographics, etiology of pancreatitis, WON size, and location. There was no difference in the technical success between the two groups $(100 \% v s$. $98.8 \%, P=1)$, or in clinical success rates $(78.7 \% v s .77 .9 \%)$. There was a significant difference in the required number of direct endoscopic necrosectomies to achieve clinical success in the PPI $v s$. non-PPI group ( $3.2 v s .4 .6$ respectively, $P<0.01)$. There were significantly more cases of stent occlusion in the non-PPI group $v s$. PPI group $(9.5 \% v s .20 .1 \% P=0.012)$, but all other documented adverse events were not significantly different. Conclusion: Discontinuing PPIs during endoscopic drainage and necrosectomy of symptomatic WON appears to reduce the number of endoscopic procedures required to achieve resolution. Continuous PPI results in higher rates of early stent occlusion.
\end{abstract}

Key words: Direct endoscopic necrosectomy, EUS, lumen-apposing metal stent, proton pump inhibitor, walled-off necrosis

\begin{tabular}{|l|l|}
\hline \multicolumn{2}{|c|}{ Access this article online } \\
\hline Quick Response Code: & Website: \\
\hline & www.eusjournal.com \\
\hline & \\
\hline
\end{tabular}

This is an open access journal, and articles are distributed under the terms of the Creative Commons Attribution-NonCommercial-ShareAlike 4.0 License, which allows others to remix, tweak, and build upon the work non-commercially, as long as appropriate credit is given and the new creations are licensed under the identical terms.

For reprints contact: reprints@medknow.com

How to cite this article: Powers PC, Siddiqui A, Sharaiha RZ, Yang G, Dawod E, Novikov AA, et al. Discontinuation of proton pump inhibitor use reduces the number of endoscopic procedures required for resolution of walled-off pancreatic necrosis. Endosc Ultrasound 2019;8:194-8.

\section{Address for correspondence}

Dr. Douglas G. Adler, Huntsman Cancer Center, University of Utah School of Medicine, 30n 1900e 4r118, Salt Lake City, Utah 84132, USA. E-mail: douglas.adler@hsc.utah.edu

Received: 2018-08-24; Accepted: 2018-10-30; Published online: 2019-02-04 
Powers, et al:: Discontinuation of PPI reduces endoscopic procedures required for resolution of WOPN

\section{INTRODUCTION}

Pancreatic fluid collections (PFCs), including walled-off necrosis (WON), commonly occur as a complication of pancreatitis and represent a therapeutic challenge for clinicians. EUS-guided drainage of WON is well established as first-line therapy, most commonly through lumen apposing metal stents (LAMS) to allow drainage of necrotic material into the stomach and access for direct endoscopic necrosectomy (DEN). The EUS-guided endoscopic approach is demonstrably as effective as surgical and percutaneous techniques but has been shown to have lower cost and lower morbidity. ${ }^{[1,2]}$

Several studies have demonstrated high technical and clinical success rates with endoscopic drainage and debridement of WON. ${ }^{[3,4]}$ While the endoscopic approach is now the first line, there still remains some uncertainty regarding ideal simultaneous medical therapy. Currently, some physicians use concurrent acid suppression therapy in the form of proton pump inhibitors (PPIs), which presumably allows a healthier communicating tract and may reduce the rates of certain adverse events, including bleeding and gastric ulceration. However, PPI use leads to less acidic gastric contents, which may retard the rate of dissolution of solid necrotic debris.

The aim of this study was to examine the use of PPI therapy in patients undergoing direct necrosectomy for the treatment of WON. This is the first study to investigate the utility of PPI therapy in these patients.

\section{METHODS}

We performed a multicenter, retrospective study of patients with WON of the pancreas who received LAMS between December 2013 and December 2016. Medical records, endoscopy reports, laboratory results, radiologic studies, and other records were reviewed for all patients included in this study.

WON was defined as per revised Atlanta classification, which was determined by cross-sectional imaging and/ or EUS. ${ }^{[5]}$ Necrosectomy and LAMS placement were performed at the discretion of the clinician. Indications for treatment included but were not limited to infected WON, gastric outlet or biliary obstruction, refractory abdominal pain, ongoing systemic illness, anorexia, or persistent weight loss. Collected data included patient demographics, cause of pancreatitis, WON size and location, and procedure details including the number of endoscopic procedures, diameter of LAMS used, and supplemental techniques.

Patients included in the study were divided into two groups: Patients on PPIs continuously during the interval of therapy, and those not on PPIs continuously during therapy. Outcome data included clinical and technical success of the procedure, number of DEN interventions required, and adverse events. The technical success was defined as completion of transmural stent placement and subsequent drainage of WON. Clinical success was defined as resolution of WON on follow-up imaging without the need for further intervention. IRB approval was obtained for this study. While individual informed consents are not required at our centers for retrospective (no cost, no risk, and no patient contact) studies such as this, institutional protocols and consent forms include patient consent to having their data used in research studies.

All values are presented as mean, median (range), or percentage. The primary outcomes of this study were to evaluate the technical success and clinical outcomes between the PPI and non-PPI groups. Secondary outcomes were patient adverse events and the number of endoscopic necrosectomy session required to achieve clinical success in the two groups. Data were analyzed using cross tabulation. Categorical variables were evaluated using Mantel-Haenszel Chi-Square or Fisher's exact test, where appropriate. Continuous data were compared using the unpaired $t$-test or Mann-Whitney tests. All values were presented as means \pm standard deviation Statistical significance was determined a priori at $P \leq 0.05$. Stepwise logistic regression was performed using SAS V9.1 (SAS Institute, Cary, NC, USA).

\section{Endoscopic technique}

All endoscopic procedures were performed by experienced endoscopists. Broad spectrum antibiotics were used intraprocedurally and for 3-5 days following the procedure. All endoscopic drainage procedures were performed transmurally using a LAMS. A linear array EUS was used to locate the WON collection and identify an adequate site of the puncture. If a first-generation LAMS system was used, cyst puncture was generally conducted using the Seldinger technique, using a balloon dilator to dilate the tract to 4-6 mm. A LAMS delivery system (AXIOSTM, Boston Scientific, Natick, MA, USA) was then advanced over the guidewire and into the 
Powers, et al:: Discontinuation of PPI reduces endoscopic procedures required for resolution of WOPN

cavity. In cases where second-generation electrocautery enhanced delivery system was used (Hot AXIOSTM, Boston Scientific, Natick, MA, USA), the LAMS catheter advanced endoscopically into the WON cavity under EUS guidance while current was applied to the diathermic tip of the catheter.

Endoscopic transmural necrosectomy was performed on either a scheduled basis or as clinically indicated, at the discretion of the performing endoscopist. Necrosectomy could be performed immediately following endoscopic transmural drainage or delayed 1-2 weeks to allow tract maturity. During necrosectomy, a forward-viewing gastroscope was advanced into the WON cavity through the LAMS, and the cavity was then irrigated. Nasocystic drains were used to irrigate the WON collection at the discretion of the endoscopist.

\section{RESULTS}

Patient demographics and walled off necrosis procedure characteristics

A total of 272 patients who underwent successful EUS-guided transmural drainage of WON using LAMS from 2013 to 2016 at 8 centers were included in this study. 136 patients used PPIs continuously during the therapy (PPI group), and 136 patients were not on PPIs during the interval of therapy (non-PPI group). The PPI and non-PPI groups were similar with regards to age, gender, etiology of pancreatitis, cyst size, and location [Table 1]. The mean patient age was 49.1-year-old, and the most common underlying etiology of pancreatitis was gallstones $(37.8 \%)$ followed by alcohol $(24.2 \%)$. The WON collections were located in the pancreatic head in $15.1 \%$ patients and the body/tail in $84.9 \%$ patients [Table 1].

With regards to stent placement, 231 patients underwent transgastric LAMS placement while 41 patients underwent transduodenal placement [Table 2]. The diameters of the LAMS stents (either $10 \mathrm{~mm}$ or $15 \mathrm{~mm}$ ) used for transmural WON drainage were similar for the PPI and non-PPI groups $(P=0.19)$ [Table 2]. Disconnected pancreatic duct syndrome was noted in $11.7 \%$ of patients resulting in ERCP with PD stent placement.

\section{Technical success and clinical outcomes}

Technical success, as defined by successful access and drainage of WON through the placement of a LAMS, was not statistically different between the PPI and non-PPI groups $(100.0 \%$ vs. $98.8 \%$ respectively; $P=0.21)$ [Table 3]. Clinical success of the procedure was defined as complete resolution of the WON on follow-up imaging, and no further endoscopic procedure required. The PPI and non-PPI groups had similar clinical success rates $(78.7 \%$ and $77.9 \%$ respectively, $P=0.88)$. However, to achieve this clinical success, the two groups required significantly different numbers of DEN procedures. Not including the initial LAMS placement, the PPI group required a median of 4.6 procedures, compared to 3.2 in the non-PPI group $(P<0.01)$. The PPI group had $14.0 \%$ achieve

Table 1. Patient demographics and walled off necrosis characteristics

\begin{tabular}{lcc}
\hline & PPI group (\%) & Non-PPI group (\%) \\
\hline $\begin{array}{l}\text { Mean age (years) } \\
\text { Gender }\end{array}$ & 48.4 & 51.5 \\
Female & $44(32.4)$ & $52(38.2)$ \\
Male & $92(67.6)$ & $84(61.8)$ \\
Pancreatitis etiology & & \\
Gallstone & $46(33.8)$ & $57(41.9)$ \\
Alcohol & $30(22.1)$ & $36(26.5)$ \\
Idiopathic & $25(18.4)$ & $16(11.8)$ \\
Trauma & $15(11.0)$ & $12(8.8)$ \\
Other & $24(17.6)$ & $15(11.0)$ \\
WON location & & \\
Pancreatic head & $22(16.2)$ & $19(14.0)$ \\
Pancreatic body/tail & $114(83.8)$ & $117(86.0)$ \\
Mean WON long-axis (mm) & 116.2 & 122.6 \\
\hline PPI: Proton pump inhibitors, WON: Walled off necrosis &
\end{tabular}

Table 2. Walled off necrosis procedure characteristics

\begin{tabular}{lccc}
\hline & PPI group (\%) & Non-PPI group (\%) & $P$ \\
\hline $\begin{array}{l}\text { Site of } \\
\text { cyst-enterostomy }\end{array}$ & $22(16.2)$ & $19(14.0)$ & 0.9 \\
$\quad \begin{array}{l}\text { Stomach } \\
\quad \text { Duodenum }\end{array}$ & $114(83.8)$ & $117(86.0)$ & \\
$\begin{array}{l}\text { Axios diameter }(\mathrm{mm}) \\
10\end{array}$ & $14(10.3)$ & $7(5.1)$ & 0.19 \\
15 & $122(89.7)$ & $129(94.8)$ & \\
$\begin{array}{l}\text { Nasocystic tube } \\
\text { placement }\end{array}$ & 36 & 31 & 0.57 \\
\hline PPI: Proton pump inhibitors & & &
\end{tabular}

Table 3. Direct endoscopic necrosectomies procedure outcomes

\begin{tabular}{lccc}
\hline & PPI group $(n=136)$ & Non-PPI group $(n=136)$ & $P$ \\
\hline $\begin{array}{l}\text { Technical } \\
\text { success (\%) }\end{array}$ & 100.0 & 98.8 & 1 \\
$\begin{array}{l}\text { Clinical } \\
\text { success (\%) }\end{array}$ & 78.7 & 77.9 & 0.88 \\
$\begin{array}{l}\text { Mean DEN } \\
\text { sessions }\end{array}$ & 4.6 & 3.2 & $<0.01$ \\
\hline
\end{tabular}

DEN: Direct endoscopic necrosectomies, PPI: Proton pump inhibitors 
Powers, et al:: Discontinuation of PPI reduces endoscopic procedures required for resolution of WOPN

resolution after just the initial LAMS placement without requiring subsequent procedures, compared to $22.1 \%$ in the non-PPI group, although this was not statistically significant $(P=0.14)$.

\section{Adverse events}

Overall, the two groups had similar rates of adverse events. The most common adverse events noted in both groups were stent occlusion requiring endoscopic clearance during subsequent necrosectomy $(15.0 \%)$, infection $(8.8 \%)$, stent migration $(4.7 \%)$, and bleeding (4.4\%) [Table 4]. When comparing the PPI $v$ s. non-PPI groups, stent occlusion occurred significantly more in the non-PPI group ( $9.5 \%$ vs. $20.1 \% . P=0.012)$. Bleeding rates were similar between PPI and non-PPI groups ( $3.7 \%$ vs. $5.1 \%$ respectively, $P=0.79)$, as were infection rates $(5.8 \%$ vs. $11.7 \% P=0.13)$ and stent migration $(7.4 \%$ vs. $2.2 \%, P=0.056)$.

\section{DISCUSSION}

The use of LAMS is an effective technique for resolving PFCs, including WONs. ${ }^{[6-8]}$ While surgical and percutaneous approaches have their place in refractory cases, the convenience and safety of an endoscopic approach have made it the preferred first-line therapy for $\mathrm{WON}$, and this practice has been supported by large, randomized controlled trials. ${ }^{[9-12]}$ Several groups have demonstrated high technical and clinical success rates. ${ }^{[13-19]}$ However, despite the growing number of physicians using LAMS drainage for WONs, the ideal concomitant medical therapy remains unknown. In this study, we aimed to investigate how the concurrent use of acid suppression impacted the efficacy and adverse event rates in LAMS for WONs.

Physiologically, arguments for both strategies can be made. Continued production of stomach acid which should, in theory, enter into the WON may promote dissolution of solid necrotic debris, allow solid contents to more easily pass through the LAMS spontaneously to the stomach, and reduce the need for endoscopic necrosectomy procedures. This should also theoretically reduce the amount of devitalized

Table 4. Adverse events

\begin{tabular}{lcccc}
\hline & $n(\%)$ & PPI group & Non-PPI group & $P$ \\
\hline GI bleeding & $12(4.4)$ & 5 & 7 & 0.79 \\
Infection & $24(8.8)$ & 8 & 16 & 0.13 \\
Stent occlusion & $41(15.0)$ & 13 & 28 & 0.012 \\
Stent migration & $13(4.7)$ & 10 & 3 & 0.056 \\
\hline \multicolumn{4}{l}{ PPI: Proton pump inhibitors, GI: Gastrointestinal }
\end{tabular}

PPI: Proton pump inhibitors, GI: Gastrointestinal tissue thereby improving clinical success and stent patency rates. Conversely, one could argue that the suppression of gastric acid production may yield a healthier communicating tract once LAMS has been placed. With more acidic gastric contents, there could theoretically be more adverse events such as ulceration at the cystenterostomy site and an increased risk of subsequent bleeding or even perforation.

Our study included 272 patients, all of whom received LAMS for endoscopic resolution of WON. The cohort was split into those receiving continuous PPI therapy and those without. The two groups were well matched with regards to demographics, etiology of pancreatitis, WON location, and WON size. In both PPI and non-PPI groups, the technical success rates were high $(100.0 \%$ vs. $98.8 \%$ respectively), which is comparable to prior studies investigating LAMS placement for WON. ${ }^{[8-14]}$ Similarly, the two groups did not have statistically significant differences in the rates of clinical success $(78.7 \%$ vs. $77.9 \%)$. This suggests that acid suppression therapy is not necessarily integral to the ultimate clinical success in the endoscopic treatment of WON.

Of critical importance, although technical and clinical success rates were similar in the two groups, the PPI group required significantly more DEN procedures to achieve this clinical success (4.6 vs. 3.2 procedures). To the best of our knowledge, this is the first large study to investigate the utility of acid suppression in these patients. Our study suggests that acidic gastric contents may confer a more potent clinical response in patients with WON treated through LAMS whereas continuous PPI therapy appears to decrease the per-procedure effectiveness. This could have considerable implications for future treatment. While endoscopy is the preferred method for treatment of WON, DEN procedures are not without inherent risk. These results suggest that withholding PPI therapy could potentially result in more efficient treatment of WON.

Overall, the combined adverse event rates were similar between the two groups. The non-PPI group was noted to have an overall significantly higher rate of stent occlusion; the reasons for this are unclear but may be due to more solid debris being liberated from the WON cavity. Thus, while withholding PPI during therapy would likely reduce the number of DEN, this must be weighed against the increased risk of stent occlusion. 
Powers, et al:: Discontinuation of PPI reduces endoscopic procedures required for resolution of WOPN

This is the first study to directly compare LAMS procedures with and without PPI use, and the optimization of medical therapy should continue to be investigated. The strengths of this study include the relatively large sample size, and the use of multiple centers to gather patient data. A limitation of this study was its retrospective nature. This imparts inherent heterogeneity in the data including follow-up time and variability in endoscopic technique. Further investigation with randomized prospective trials would be needed to support our results.

\section{CONCLUSION}

Overall, we have demonstrated that the use of PPI therapy in patients being treated for WON with LAMS and DEN reduces clinical efficacy and that by withholding PPI we can decrease the total number of DEN procedures required to resolve WON. PPI use does appear to have an early protective effect against the risk of stent occlusion, while the overall adverse event rates appear to be similar. The results of this study argue for the discontinuation of PPI during endoscopic therapy for WON. Large-scale randomized controlled studies into this question are warranted.

\section{Financial support and sponsorship}

Nil.

\section{Conflicts of interest}

DG Adler, T. Kowalski, A. Siddiqui, and M Kahaleh are consultants to Boston Scientific.

\section{REFERENCES}

1. Varadarajulu S, Bang JY, Sutton BS, et al. Equal efficacy of endoscopic and surgical cystogastrostomy for pancreatic pseudocyst drainage in a randomized trial. Gastroenterology 2013;145:583-900.

2. Adler DG. Surgery versus endoscopy for patients with infected pancreatic necrosis. Lancet 2018;391:6-8.

3. Gardner TB, Coelho-Prabhu N, Gordon SR, et al. Direct endoscopic necrosectomy for the treatment of walled-off pancreatic necrosis: Results from a multicenter U.S. Series. Gastrointest Endosc 2011;73:718-26.

4. Gornals JB, De la Serna-Higuera C, Sánchez-Yague A, et al. Endosonography-guided drainage of pancreatic fluid collections with a novel lumen-apposing stent. Surg Endosc 2013;27:1428-34.

5. Banks PA, Bollen TL, Dervenis C, et al. Classification of acute pancreatitis-2012: Revision of the Atlanta classification and definitions by international consensus. Gut 2013;62:102-11.

6. Binmoeller KF, Shah J. A novel lumen-apposing stent for transluminal drainage of nonadherent extraintestinal fluid collections. Endoscopy 2011;43:337-42.

7. Itoi T, Nageshwar Reddy D, Yasuda I. New fully-covered self-expandable metal stent for endoscopic ultrasonography-guided intervention in infectious walled-off pancreatic necrosis (with video). J Hepatobiliary Pancreat Sci 2013;20:403-6.

8. Khreiss M, Zenati M, Clifford A, et al. Cyst gastrostomy and necrosectomy for the management of sterile walled-off pancreatic necrosis: A comparison of minimally invasive surgical and endoscopic outcomes at a high-volume pancreatic center. J Gastrointest Surg 2015;19:1441-8.

9. Baron TH, Harewood GC, Morgan DE, et al. Outcome differences after endoscopic drainage of pancreatic necrosis, acute pancreatic pseudocysts, and chronic pancreatic pseudocysts. Gastrointest Endosc 2002;56:7-17.

10. Bakker OJ, van Santvoort HC, van Brunschot S, et al. Endoscopic transgastric vs. surgical necrosectomy for infected necrotizing pancreatitis: A randomized trial. JAMA 2012;307:1053-61.

11. Rasch S, Phillip V, Reichel S, et al. Open surgical versus minimal invasive necrosectomy of the pancreas - A retrospective multicenter analysis of the German pancreatitis study group. PLoS One 2016;11:e0163651.

12. van Brunschot S, van Grinsven J, van Santvoort HC, et al. Endoscopic or surgical step-up approach for infected necrotising pancreatitis: A multicentre randomised trial. Lancet 2018;391:51-8.

13. Jeffrey SB, Adler DG. Lumen apposing metal stents: A review of current uses and outcomes. Gastrointest Interv 2017;6:9-14.

14. Bang JY, Hasan MK, Navaneethan U, et al. Lumen-apposing metal stents for drainage of pancreatic fluid collections: When and for whom? Dig Endosc 2017;29:83-90.

15. Shah RJ, Shah JN, Waxman I, et al. Safety and efficacy of endoscopic ultrasound-guided drainage of pancreatic fluid collections with lumen-apposing covered self-expanding metal stents. Clin Gastroenterol Hepatol 2015;13:747-52.

16. Walter D, Will U, Sanchez-Yague A, et al. A novel lumen-apposing metal stent for endoscopic ultrasound-guided drainage of pancreatic fluid collections: A prospective cohort study. Endoscopy 2015;47:63-7.

17. Siddiqui AA, Adler DG, Nieto J, et al. EUS-guided drainage of peripancreatic fluid collections and necrosis by using a novel lumen-apposing stent: A large retrospective, multicenter U.S. Experience (with videos). Gastrointest Endosc 2016;83:699-707.

18. Sharaiha RZ, Tyberg A, Khashab MA, et al. Endoscopic therapy with lumen-apposing metal stents is safe and effective for patients with pancreatic walled-off necrosis. Clin Gastroenterol Hepatol 2016;14:1797-803.

19. Rinninella E, Kunda R, Dollhopf M, et al. EUS-guided drainage of pancreatic fluid collections using a novel lumen-apposing metal stent on an electrocautery-enhanced delivery system: A large retrospective study (with video). Gastrointest Endosc 2015;82:1039-46. 